\title{
Situs inversus totalis. A propósito de 2 casos clínicos
}

\author{
MARÍA JUNCOS C. ${ }^{1}$, MARÍA AMPARO ROS F. ${ }^{1}$, \\ MARÍA MARAVALL LL. ${ }^{2}$, JULIO ÁLVAREZ-PITTI ${ }^{3}$ \\ 1. Médico residente. Servicio de Pediatría. Consorcio Hospital General Universitario Valencia, España. \\ 2. Médico adjunto. Servicio de Pediatría. Sección Cardiología Pediátrica. Consorcio Hospital General Universitario. Valencia, España. \\ 3. Médico adjunto. Unidad Riesgo Cardiovascular. Consorcio Hospital General Universitario. Valencia, España.
}

\begin{abstract}
Situs inversus totalis -2 case reports

Introduction: Situs inversus totalis is a rare find and only a small percentage are associated with heart disease; its diagnosis is usually made incidentally. Objective: To discuss the diagnostic features of situs inversus totalis and the importance of early diagnosis. Case reports: Two pediatric patients aged 9 and 14 years who were incidentally diagnosed are reported. The first case presented chest pain and during cardiac auscultation, increased heart sounds were heard on the right precordium and attenuated on the left. An electrocardiogram (ECG) showed $\mathrm{P}$ wave and QRS axis equal to $+150^{\circ}$, narrow $\mathrm{QRS}$ voltage attenuated in V3 -V6 precordial leads, and negative $\mathrm{T}$ waves in leads V1-V4 and aVL. Chest radiography confirmed dextrocardia, and gastric bubble was on the right and hepatic shadow on the left. Echocardiography showed classic mirror dextrocardia without associated malformations. In the second case, dextrocardia was found incidentally after radiography was requested for the evaluation of scoliosis. ECG showed QRS of $+120^{\circ}, \mathrm{P}$ wave axis of $+150^{\circ}$ and narrow QRS voltage axis attenuated on left precordial leads. Doppler echocardiography confirmed dextrocardia without associated anomalies. Abdominal ultrasound found the liver in left upper quadrant and the spleen in right upper quadrant. Conclusions: Early diagnosis of situs inversus totalis is important because the thoracic and abdominal surgical approach is different and certain diseases could be presented with unusual characteristics. Also, after the diagnosis of situs inversus, the presence of associated pathologies such as primary ciliary dyskinesia can be studied (Kartagener syndrome).
\end{abstract}

(Key words: Dextrocardia, situs inversus, electrocardiogram, childhood, ciliary dyskinesia, Kartagener).

Rev Chil Pediatr 2014; 85 (3): 344-350

\section{RESUMEN}

Introducción: El situs inversus totalis es un hallazgo poco común. Sólo un pequeño porcentaje presentará cardiopatías asociadas, por lo que el diagnóstico suele realizarse de forma casual. Objetivo: Destacar las claves

Recibido el 28 de abril de 2013, devuelto para corregir el 17 de septiembre de 2013, segunda versión 25 de noviembre de 2013, tercera versión 24 de enero de 2014, aceptado para publicación el 28 de marzo de 2014.

Este trabajo cumple con los requisitos sobre consentimiento /asentimiento informado, comité de ética, financiamiento, estudios animales y sobre la ausencia de conflictos de intereses según corresponda.

Correspondencia a:

María Juncos C.

E-mail: mariajuncl@hotmail.com 
diagnósticas del situs inversus totalis y la importancia de un diagnóstico temprano. Caso clínico: Presentamos dos pacientes pediátricos de 9 y 14 años que fueron diagnosticados de forma incidental. El primer caso fue estudiado por dolor precordial, en la auscultación cardíaca se escuchaba aumento de ruidos cardíacos en precordio derecho y atenuados en el izquierdo. El electrocardiograma (ECG) mostró eje QRS y onda P de $+150^{\circ}$, QRS estrecho de voltaje atenuado en precordiales V3-V6 y ondas T negativas en V1-V4 y aVL. La radiografía de tórax confirmó dextrocardia, además se apreció burbuja gástrica a la derecha y sombra hepática a la izquierda. La ecocardiografía evidenció dextrocardia especular clásica sin malformaciones asociadas. En el segundo caso se encontró incidentalmente dextrocardia en radiografía solicitada para evaluación de escoliosis. El ECG objetivó eje $\mathrm{QRS}+120^{\circ}$, eje onda $\mathrm{P}+150^{\circ}$, QRS estrecho de voltaje atenuado en precordiales izquierdas. La ecocardiografía doppler confirmó dextrocardia especular sin anomalías asociadas. En ecografía abdominal el hígado se encontró en hipocondrio izquierdo y el bazo en hipocondrio derecho. Conclusión: El diagnóstico temprano del situs inversus totalis es importante, dado que el abordaje quirúrgico torácico y abdominal es diferente y determinadas patologías pueden presentarse con clínica inusual. Además, tras el diagnóstico de situs inversus se puede estudiar la presencia de patologías asociadas como discinesia ciliar primaria (Síndrome de Kartagener).

(Palabras clave: Dextrocardia, situs inversus, electrocardiograma, infancia, discinesia ciliar, Kartagener).

Rev Chil Pediatr 2014; 85 (3): 344-350

\section{Introducción}

El situs inversus totalis se caracteriza por la posición invertida de los órganos torácicos y abdominales con respecto al plano sagital como resultado de anomalías en la rotación del tubo cardíaco durante el período embrionario ${ }^{1}$. Es una entidad poco frecuente (prevalencia estimada de 1/10.000 nacimientos) cuyo mecanismo es desconocido, aunque, recientemente, han sido identificados numerosos genes implicados en la aparición de defectos en la asimetría izquierda-derecha ${ }^{2,3}$. Tiene un patrón de herencia autosómico recesivo con igual incidencia en ambos sexos. En la edad pediátrica con frecuencia el diagnóstico se realiza de forma casual, dada la ausencia de síntomas, ya que sólo un 5-10\% asocian malformaciones cardiovasculares congénitas ${ }^{4}$.

El estudio de la lateralidad de los órganos en el feto es parte del examen ultrasonográfico $^{5}$ rutinario, por lo cual a pesar de su baja frecuencia se debe tener en cuenta la posibilidad de la existencia de situs inversus durante la realización de esta prueba. Las avanzadas técnicas actuales de ultrasonidos y la experiencia médica que se ha ido acumulando desde la existencia de la ecocardiografía fetal vía transabdominal materna, ha permitido aumentar la sensibilidad y especificidad de dicha técnica, consiguiendo mayor detección de cardiopatías prenatales $^{6}$.

El objetivo de esta comunicación es describir dos casos clínicos para revisar las claves diagnósticas de esta entidad y resaltar la importancia de su diagnóstico temprano.

\section{Caso 1}

Varón de 9 años, sin antecedentes patológicos de interés, remitido a servicio de urgencias por dolor precordial intermitente de 1 mes de evolución, asociado a alteración en el electrocardiograma (ECG): atenuación del QRS e inversión de onda $\mathrm{T}$ en derivaciones precordiales. No refería palpitaciones, síntomas vegetativos ni episodios sincopales.

A la exploración física presentaba buen aspecto general, manteniéndose hemodinámicamente estable, destacando dolor a la palpación a nivel de la musculatura torácica izquierda que aumentaba con la inspiración y la movilización de miembros superiores. En la auscultación cardíaca se escuchaba aumento de ruidos cardíacos en precordio derecho y atenuados en el izquierdo.

Dado el tiempo de evolución del dolor y la alteración electrocardiográfica referida, se repitió ECG, persistiendo eje QRS y onda $\mathrm{P}$ de $+150^{\circ}$, QRS estrecho de voltaje atenuado en precordiales V3-V6 y ondas T negativas en V1-V4 y aVL (figura 1).

En la radiografía de tórax se confirmó la 


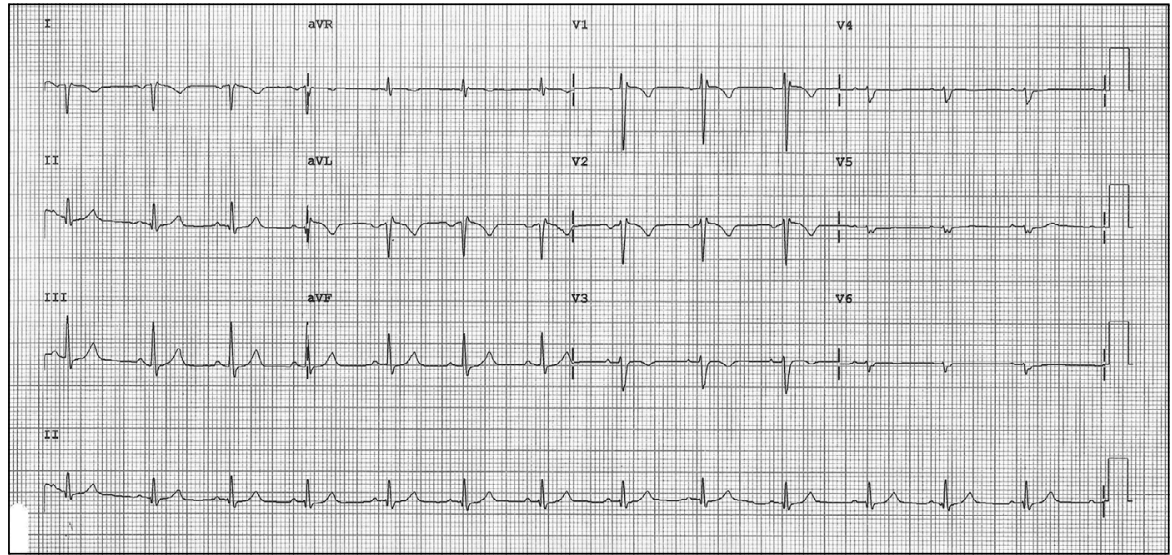

Figura 1. Electrocardiograma donde se observa eje del QRS y de la onda $\mathrm{P}$ de $+150^{\circ}$, QRS estrecho de voltaje atenuado en precordiales $\mathrm{V} 3-\mathrm{V} 6$ $\mathrm{y}$ ondas $\mathrm{T}$ negativas en V1-V4 y aVL. presencia de dextrocardia, además se apreció burbuja gástrica a la derecha y sombra hepática a la izquierda, por lo que se sospechó situs inversus, que se confirmó en ecografía abdominal (figura 2).

El paciente fue remitido a Cardiología Infantil donde se realizó ecocardiografía evidenciándose dextrocardia especular clásica $\sin$ malformaciones cardíacas asociadas (figuras $3,4)$.

Ante la sospecha de etiología músculo-esquelética, el dolor torácico se trató con antiinflamatorios con mejoría progresiva, encontrándose asintomático en el momento de acudir a control.

\section{Caso 2}

Varón de 14 años, con antecedente de convulsiones febriles en la primera infancia, remitido a Cardiología Infantil al objetivarse de forma casual presencia de dextrocardia en radiografía solicitada para evaluación de escoliosis (figura 5). No refería clínica cardiológica.

En el ECG se objetivó eje QRS $+120^{\circ}$, eje onda $\mathrm{P}+150^{\circ}$, QRS estrecho de voltaje atenuado en precordiales izquierdas, compatible con el hallazgo radiológico (figura 6). Se confirmó dextrocardia especular sin anomalías asociadas en ecocardiografía doppler. En ecografía abdominal el hígado se encontró de características normales localizado en hipocondrio izquierdo con vías biliares normales y el bazo de aspecto normal localizado en hipocondrio derecho, todos ellos hallazgos compatibles con situs inversus totalis sin alteraciones en los flujos vasculares. No se objetivó poliesplenia.

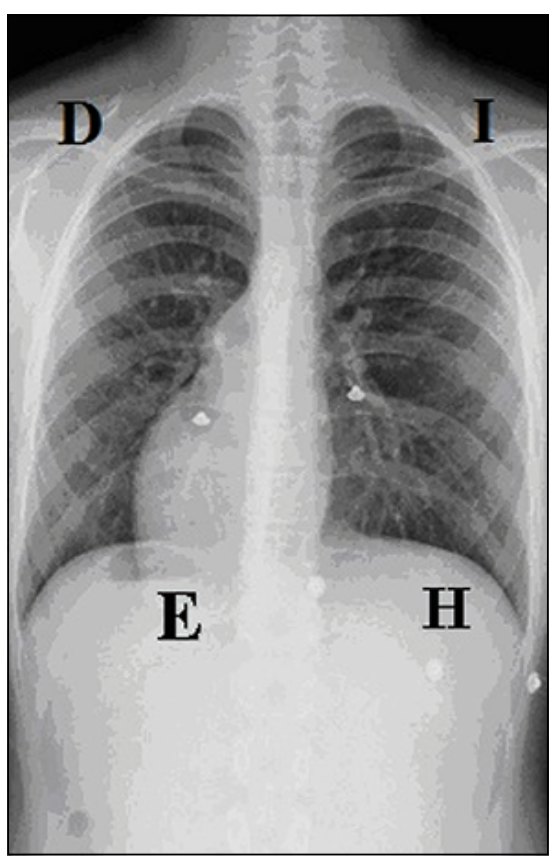

Figura 2. Radiografía anteroposterior de tórax en la que se observa la silueta cardíaca localizada en el hemitórax derecho, con el ápex en la misma dirección, se observa la cámara gástrica (E) con contenido aéreo en la región subdiafragmática derecha, y la sombra hepática $(\mathrm{H})$ en la región subdiafragmática izquierda. 


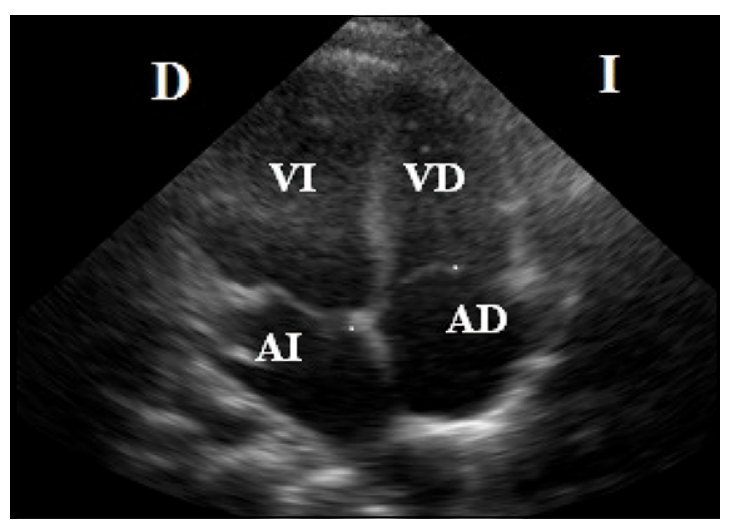

Figura 3. Ecocardiograma transtorácico en plano de cuatro cámaras en el que se observa: la aurícula y el ventrículo anatómicamente derechos (AD, VD), situados en el lado izquierdo. La aurícula y ventrículo anatómicamente izquierdos (AI, VI), situados en el lado derecho. Permite observar la desembocadura de las venas pulmonares, conservando la relación anatómica y sin presentar ninguna malformación asociada.

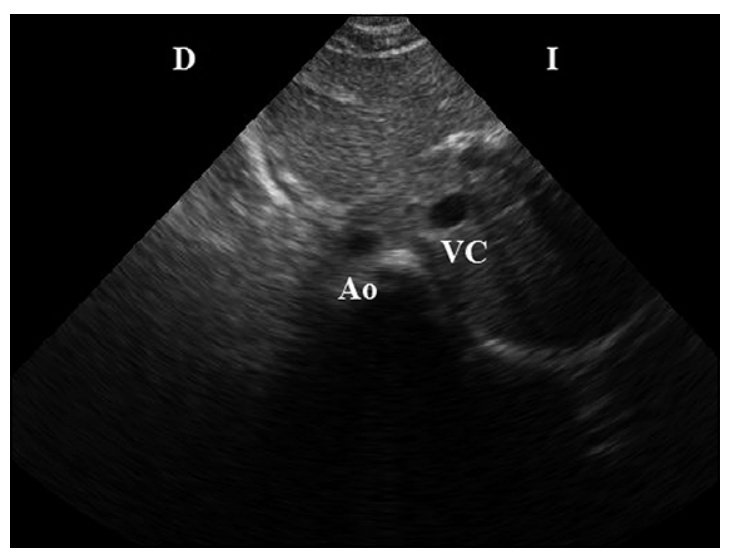

Figura 4. Ecocardiograma transtorácico en proyección subcostal en la que se observa el situs inversus abdominal, con la aorta (Ao) a la derecha y la vena cava (VC) a la izquierda.

\section{Discusión}

En la especie humana el 99,99\% de los individuos desarrollan durante la etapa embrionaria una asimetría izquierda-derecha de los órganos toracoabdominales denominada situs solitus en la que encontramos: hacia el lado izquierdo un pulmón bilobulado, el ápex cardíaco, estómago y bazo y hacia la derecha un pulmón trilobulado, la vena cava, el apéndice cecal y un lóbulo hepático mayor?

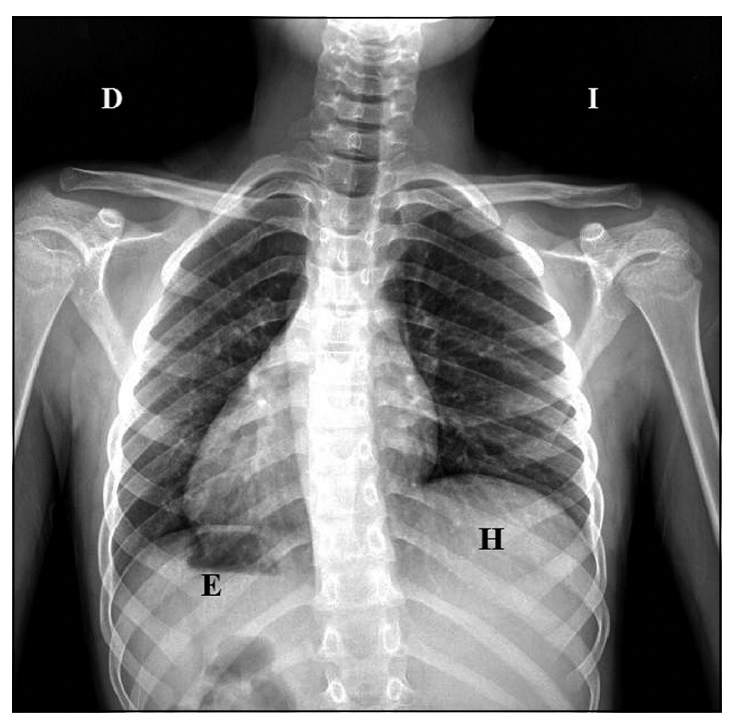

Figura 5. Radiografía anteroposterior de tórax en la que se observa la silueta cardíaca localizada en el hemitórax derecho, con el ápex en la misma dirección, se observa la cámara gástrica (E) con contenido aéreo en la región subdiafragmática derecha, y la sombra hepática $(\mathrm{H})$ en la región subdiafragmática izquierda.

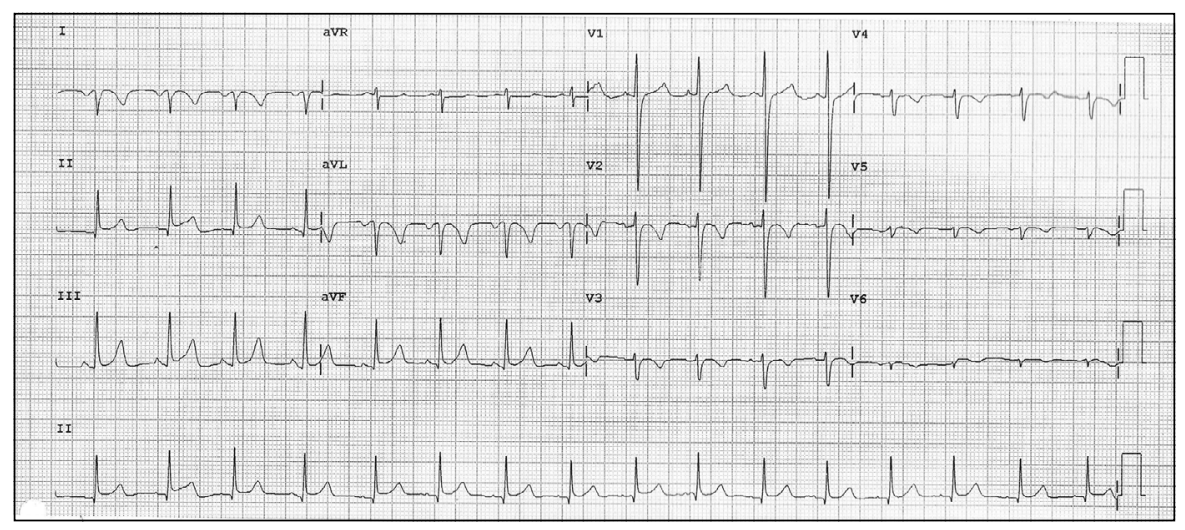

Figura 6. Electrocardiograma donde se observa eje del QRS $+120^{\circ}$ y de la onda P de $+150^{\circ}$, QRS estrecho de voltaje atenuado en precordiales V4-V6 y ondas T negativas en V2-V4 y aVL. 
Cuando existe inversión completa de los órganos lateralizados ("imagen en espejo") estamos ante una situación de situs inversus totalis ${ }^{2}$, hallazgo descrito por primera vez en 1788 por Matthew Baillie ${ }^{8}$. Este hecho ocurre en torno a 1/10.000 nacimientos. Entre ambos extremos de situs solitus y situs inversus se encuentra el espectro del situs ambiguus, caracterizado por isomerismo auricular y múltiples malformaciones en órganos torácicos o abdominales 9 .

Sólo un $5-10 \%$ de los pacientes con situs inversus presentan cardiopatías asociadas y malformaciones cardiovasculares debutando con síntomas atribuibles a estas patologías. Entre las más frecuentes se encuentran la transposición de los grandes vasos, comunicación interauricular, comunicación interventricular y retorno venoso anómalo ${ }^{10}$. El resto de los pacientes se encuentran asintomáticos y la dextrocardia suele suponer un hallazgo casual en la exploración física o radiografía de tórax de rutina tal y como ocurre en los casos clínicos presentados.

Las mejoras y avances en ecocardiografía fetal así como la mayor experiencia, han conducido en los últimos años a un diagnostico prenatal preciso y fiable de cardiopatías congénitas. Así mismo, mediante la ecografía prenatal podría diagnosticarse el situs inversus en la etapa fetal. En la exploración ecográfica transabdominal, los métodos habituales de orientación espacial, se basan en varios parámetros incluyendo la posición maternal, la posición fetal y sus órganos abdominotorácicos junto con la orientación del transductor ${ }^{11}$, por lo que en ocasiones la diferenciación de la lateralidad derecha-izquierda en el feto puede ser difícil y confusa. Este hecho hace que el diagnóstico de situs inversus o dextrocardia pueda retrasarse incluso hasta la edad adulta en pacientes asintomáticos, siendo mucho más precoz en aquellos casos que asocian defectos cardíacos estructurales, detectándose ya en el periodo prenatal o neonatal ${ }^{12}$. Se requiere por tanto una estandarización en los métodos de adquisición y presentación de imágenes que permita la determinación exacta del eje fetal derecha-izquierda por referencias anatómicas identificadas fácilmente ${ }^{13}$.
Además de una exploración física sugestiva y radiografía de tórax, son necesarias otras exploraciones complementarias para realizar el diagnóstico completo de situs inversus totalis. En la exploración física el latido del ápex se palpa en el lado derecho y los ruidos cardíacos o soplos se auscultan con mayor intensidad en el hemitórax derecho. Se requiere una ecocardiografía sistemática para establecer el situs, las relaciones auriculoventriculares y ventriculoarteriales, definir los drenajes sistémicos y pulmonares, y a la vez descartar patología cardíaca estructural, y una ecografía abdominal que detecte posibles malformaciones tales como hernia diafragmática, asplenia o poliesplenia $^{14}$.

El ECG puede servir para localizar las aurículas y los ventrículos. En cuanto a la localización de la aurícula, el nodo sinusal se localiza siempre en la aurícula derecha (AD), por tanto se puede utilizar el eje de la onda $P$ para localizar las aurículas. La AD está localizada en el lado opuesto al eje $\mathrm{P}^{15}$ :

- Si P está entre 0 y $+90^{\circ}$, la AD está situada en el lado derecho.

- Si P está entre $+90^{\circ} \mathrm{y}+180^{\circ}$, la AD está situada en lado izquierdo.

El método electrocardiográfico para localizar el ventrículo se basa en la despolarización del septo ventricular desde el ventrículo izquierdo (VI) al ventrículo derecho (VD). El VI suele estar situado en el mismo lado que las derivaciones precordiales que muestran ondas $\mathrm{Q}^{15}$.

- Si V6 muestra onda Q, el VI está situado a la izquierda.

- Si V1 muestra onda Q el VI está situado a la derecha.

La posición del corazón en el lado derecho del tórax puede también sospecharse en un electrocardiograma de superficie por una progresiva disminución del complejo QRS de V1 a V6, como se observa en la figura 1.

Por otra parte, cabe destacar que, los pacientes que asocien dextrocardia o situs inversus a rinosinusitis crónica, otitis media de repetición o problemas respiratorios desde el nacimiento, deben hacernos sospechar una 
discinesia ciliar primaria, ya que el $20-25 \%$ de los pacientes con situs inversus padecen una enfermedad autosómica recesiva, Síndrome de Kartagener, que asocia discinesia ciliar ${ }^{7,16}$. El diagnóstico temprano de estos pacientes puede disminuir la morbilidad, instaurándose de forma precoz tratamientos que mantengan una función pulmonar adecuada el mayor tiempo posible. Por este motivo son necesarios estudios en base a los que se puedan establecer algoritmos que orienten en la búsqueda activa de alteraciones de la motilidad ciliar. En el estudio de Busquets et al, en un paciente neonatal se llegó al diagnóstico de discinesia ciliar tras hallazgo casual de situs inversus total, al incluirse éste como criterio clínico para la realización de pruebas de cribado como la prueba de la sacarina o detección del óxido nítrico nasal y exhalado ${ }^{17}$.

\section{Conclusiones}

El situs inversus totalis suele suponer un hallazgo casual dado que la mayoría de los pacientes se encuentran asintomáticos. En la actualidad los avances en las técnicas de ecografía prenatal facilitan que pueda realizarse un diagnóstico fetal especialmente en los casos con cardiopatías asociadas ${ }^{18}$. Pese a que todavía hacen falta más estudios, ya se están viendo resultados óptimos en detección y concordancia diagnóstica pre y posnatal ${ }^{19}$.

Además de una exploración física sistemática donde se escuchen los tonos cardíacos en el hemitórax derecho, un electrocardiograma con hallazgos sugestivos de dextrocardia como son: ejes de $\mathrm{P}$ y QRS entre $+120^{\circ} \mathrm{y}+150^{\circ}$, voltaje atenuado del QRS en precordiales izquierdas y ondas $\mathrm{T}$ negativas en precordiales derechas, puede orientarnos en el diagnóstico inicial de situs inversus. Para el diagnóstico completo además, son necesarias la radiografía de tórax, ecografía abdominal y ecocardiografía para detectar posibles cardiopatías estructurales asociadas y definir las relaciones auriculoventriculares, ventriculoarteriales y los drenajes sistémicos y pulmonares.

A pesar de que los pacientes con situs inversus totalis tienen una vida similar a la po- blación general, un diagnóstico temprano de esta entidad es importante, dado que el abordaje quirúrgico torácico y abdominal es diferente en estos pacientes ${ }^{20}$, y determinadas patologías como la apendicitis, hepatitis, rotura esplénica, colecistitis o infarto agudo de miocardio se presentan en forma atípica, con los signos y síntomas propios de la afección en el lugar opuesto al esperado ${ }^{21,22}$. Por otra parte, a partir del diagnóstico de situs inversus se podría estudiar la presencia de entidades asociadas como el Síndrome de Kartagener, dados los beneficios que una detección precoz supone en la mejora de la calidad de vida de estos pacientes.

\section{Referencias}

1.- Dellamea MA, Sánchez LM, Cupelli J: Correlación anatomoradiológica del Situs Inversus Totalis. Rev Postgrado via Cátedra Med 2009; 106: 16-9.

2.- João Marta M, Menezes L, Saavedra JA, Ravara L: Um Caso de Situs Inversus Total. Rev Port Cardiol 2003; 22 (1): 91-104.

3.- Carrillo R, Arias CR, Huacuja RR, García M, Carrillo $C A$, Carrillo DM: Situs Inversus Totalis. Med Int Mex 2012; 28 (2): 187-91.

4.- Maldjian PD, Saric M: Approach to dextrocardia in adults. Am J Roentgenol [en línea] 2007 Jun [fecha de acceso 3 de noviembre de 2009]; 188 (6): 39-49.

5.- Bronshtein M, Gover A, Zimmer EZ: Sonographic definition of the fetal situs. Obstet Gynecol 2002; 99 (6): 1129-30.

6.- Ozkutlu S, Ayabakan C, Karagbz T, et al: Prenatal echocardiographic diagnosis of congenital heart disease: comparison of past and current results. Turk J Pediatr 2005; 47: 232-8.

7.- Aylsworth AS: Clinical aspects of defects in the determination of laterality. Am J Med Genet 2001; 101 (4): 345-55.

8.- Wilhelm A, Holbert JM: Situs inversus imaging. Last updated February 2009, emedicine from: htto://emedicine.medscape.com/article/413679-overview\#showall

9.- Paublo M, Bustos JC, Ramírez P: Diagnóstico prenatal de situs inversus totalis. Rev Chil Obstet Ginecol 2002; 67 (6): 494-7.

10.- Libby P, Bonow RO, Mann DL, Zipes DP: Braunwald's Heart Disease: A Textbook of Cardiovascular Medicine, 8th Ed: Saunders Elsevier, Philadelphia 2008; 10.

11.- Ozkutlu S, Bostan OM, Deren O, et al: Prenatal echocar- 
diographic diagnosis of cardiac right/left axis and malpositions according to standardized Cordes technique. Anadolu Kardiyol Derg 2011; 11: 131-6.

12.- Evans WN, Acherman RJ, Collazos JC, et al: Dextrocardia: Practical Clinical Points and Comments on Terminology. Pediatr Cardiol (2010) 31: 1-6.

13.- Cordes TM, O'Leary PW, Seward JB, Hagler DJ: Distinguishing right from left: a standardized technique for fetal echocardiography. J Am Soc Echocardiogr 1994; 7 (1): 47-53.

14.- Chitayat D, Lao A, Wilson D, et al: Prenatal diagnosis of asplenia/polysplenia syndrome. Am J Obstet Gynecol 1988; 158: 181.

15.- Myung K. Park: Cardiopatía Pediátrica. Capítulo 16. Localización de la cámara y posición cardíaca anómala.

16.- Hinojos L, Alejandre A, Garrido C, Maldonado B: Discinesia ciliar primaria como causa de infecciones recurrentes en pediatría. Informe de un caso y revisión de la literatura. Acta Pediatr Mex 2009; 30 (5): 271-4.
17.- Busquets RM, Caballero-Rabasco MA, Velasco M, Lloreta J, Garcia-Algar O: Discinesia ciliar primaria: criterios clínicos de indicación de estudio ultraestructural. Arch Bronconeumol 2013; 49 (3): 99-104.

18.- Bernasconi A, Azancot A, Simpson JM, Jones A, Sharland $G K$ : Fetal dextrocardia: diagnosis and outcome in two tertiary centres. Heart 2005; 91 (12): 1590-4.

19.- Prats $P$, Ferrer Q, Rodríguez MA, Comas C: Diagnóstico prenatal y evolución de cardiopatías congénitas. Diagn prenat 2011; 22 (4): 128-35.

20.- Adeyekun AA, Onunu AN, Mazell FO: Dextrocardia with situs inversus. A case report. West Afr J Med 2003; 22: 358-60.

21.- Urquia H, Discua L, Valenzuela R: Situs Inversus Totalis y Cardiopatía congénita: Diagnóstico casual en un lactante con neumonía. CIMEL 2010; 15 (2): 85-88.

22.- Khalil S, Kamal A, Ahmed S: Acute myocardial infarction in a patient with dextrocardia and situs inversus. $\mathrm{J}$ Electrocardiol 2005; 38: 412-3. 